\title{
Escalas de Predicción e Infección de Sitio Quirúrgico en 15625 Cirugías 2001-2003
}

\author{
HUGO A. FAJARDO RODRÍGUEZ, JEANETTE QUEMBA \\ GORDILLO y JAVIER ESLAVA SCHMALBACH
}

Recibido 30 Septiembre 2004/Enviado para Modificación 19 Diciembre 2004/Aceptado 14 Enero 2005

\section{RESUMEN}

Objetivo La infección quirúrgica es una causa importante de morbimortalidad, constituye el $25 \%$ del total de infecciones nosocomiales. A través de un programa de vigilancia intensificada y seguimiento telefónico nos propusimos detectar la infección de sitio quirúrgico en cirugías ambulatorias.

Métodos Se tomaron los pacientes en cohorte prospectiva que se intervinieron quirúrgicamente en la Clínica Carlos Lleras de Bogotá, adscrita a los Seguros Sociales, entre agosto de 2001 y febrero de 2003, los cuales tenían los siguientes criterios de inclusión: cirugía hospitalaria o ambulatoria, formato de predicción de riesgos completo y seguimiento telefónico. Se aplicaron los criterios de infección del CDC.

Resultados En total fueron 15625 pacientes, siendo principalmente de cirugía general y oftalmología. En el método de predicción SENIC, la variable cirugía abdominal fue la más frecuente y de mayor sensibilidad, mientras que en el NNIS, el mejor predictor fue cirugía prolongada. Se detectaron 69 pacientes con infección de sitio quirúrgico, para una incidencia de 0.43 infectados por cada 100 pacientes intervenidos; es la segunda causa de infección nosocomial en nuestra institución con el 19,2\%. El aislamiento de gérmenes se logró en 47 casos, siendo el más frecuente $E$. coli $(14,9 \%)$.

Conclusión El programa ha surtido benéficos efectos a sus usuarios identificando los factores de riesgo y la presencia temprana de infección posquirúrgica, disminuyéndola en un $25 \%$.

Palabras Clave: Infección, quirófano, complicaciones postoperatorias, infección hospitalaría (fuente: DeCS, BIREME).

Prediction scales and infection on surgical sites in 15625 surgeries. 2001-2003 
Objective Surgical infection is an important cause of morbidity and mortality; it accounts for $25 \%$ of nosocomial infections. Through a program of intensified monitoring and telephone follow-up we sought to detect infections in the surgical site in ambulatory surgeries.

Methods A prospective cohort of patients who underwent surgery at the Carlos Lleras Hospital in Bogotá, belonging to the Social Security Institute, between August 2001 and February 2003 was studied. Inclusion criteria were: hospital or ambulatory surgery, completion of the survey of risk prediction and telephone follow-up. The CDC criteria for nosocomial infections were applied.

Results 15625 patients were studied, being mainly of general surgery and ophtalmology. In the method of prediction SENIC, the variable abdominal surgery was the most frequent one and had the greatest sensitivity, whereas in the NNIS the best predicting variable was prolonged surgery. 69 patients with infection of surgical were detected yielding a 0,43 incidence of infection per 100 patients; surgery is the second cause of nosocomial infection in our institution accounting for $19,2 \%$ of cases. Bacterial isolation of germs was obtained in 47 cases; the most frequently identified bacteria was $E$. coli $(14,9$ $\%)$.

Conclusion The program has had beneficial effects for its users identifying the risk and the early presence of post-surgical, diminishing it in a $25 \%$.

Keys Words: Infection, operating room, postoperative complications, cross infection (source: $\mathrm{MeSH}, \mathrm{NLM}$ ).

$\mathrm{E}$ vitar la infección del sitio operatorio ha sido preocupación permanente del personal de salud. A pesar de los conocimientos sobre la técnica quirúrgica, la patogénesis de la infección del sitio operatorio y el uso de antibióticos profilàcticos, la infección quirúrgica constituye una causa importante de la morbimortalidad, aumento de la estancia hospitalaria y de los costos de atención de los pacientes sometidos a procedimientos quirúrgicos. Constituye el $25 \%$ del total de infecciones nosocomiales.(1).

En 1970 el CDC estableció el National Nosocomial Infection Surveillance Systems (NNISS), que monitoreó el rumbo de la infección quirúrgica en los hospitales de Estados Unidos. El NNISS, mostró que la infección del sitio operatorio (ISO), es la tercera infección nosocomial más frecuentemente encontrada en los pacientes hospitalizados, con una prevalencia del 14 al 16\%. En 1980 Cruse estimó que las infecciones en el sitio operatorio incrementaban entre 7,3 a 10 días la estancia hospitalaria, adicionando en promedio US \$2 000 a \$ 3152 los costos de atención. $(2,3,4)$. 
Dado que los estudios muestran que si se determinan tempranamente los riesgos, por métodos de predicción clasificando a los pacientes desde el preoperatorio, y contactándolos telefónicamente en busca de sintomatología de infección en el post operatorio, se podría disminuir las complicaciones por infección generando en los pacientes menor incapacidad y menores costos para la instituciones de salud. El grupo de vigilancia epidemiológica de la clínica Carlos Lleras Restrepo se propuso realizar este tipo de vigilancia a los pacientes de cirugía programada en forma prospectiva para

detectar la infecciòn y su relaciòn con los sistemas predictivos de riesgo NNISS y el SENIC (Study on the efficacy of nosocomial infection control). $(1,5,6,7)$.

\section{MATERIALES Y MÉTODOS}

Se tomaron los pacientes en cohorte prospectiva que se intervinieron quirúrgicamente en la Clínica Carlos Lleras de Bogotá, adscrita a los Seguros Sociales, entidad de segundo nivel de complejidad entre Agosto de 2001 y Febrero de 2003, los cuales tenían los siguientes criterios de inclusión: cirugía hospitalaria o ambulatoria, formato de predicción de riesgos completo y seguimiento telefónico. Se excluyeron los pacientes a los que se les canceló el procedimiento quirúrgico.

Los datos fueron recogidos en un formato donde se registró la información demografica y los factores de riesgo, clasificación SENIC y NNISS, seguimiento telefónico y complicaciones, secuelas o mortalidad asociada. Las variables predictoras fueron: tipo de herida, duración de la cirugía, clasificación ASA (Americam society of anesthesiologists), tipo de intervención, cirujano, factores de riesgo y comorbilidad; las variables de desenlace fueron: estancia, infección sitio quirúrgico, uso de amtimicrobianos, cultivos positivos, rehospitalización y mortalidad.

La definición de caso se aplicó según los criterios del CDC de Atlanta (Centers of disease control and prevention) para infección de herida quirúrgica así: superficiales cuando comprometen piel y tejido subcutáneo, profundas, músculo y fascias y órgano o espacio profundo cercano a la incisión. En general, se consideró infección cuando existió drenaje purulento en el sitio de incisión, signos locales de infección, aislamiento de microorganismos obtenidos en cultivo de incisión y a criterio del cirujano. (1). A los pacientes se les clasificó la incisión de acuerdo al grado de contaminación: limpia, limpia- contaminada, contaminada y sucia. En el sistema SENIC, se identificaron como factores de riesgo, herida contaminada o sucia, operación abdominal, duración de la intervención mayor de dos horas y tres o más diagnós- 
ticos asociados al egreso. En el NNISS, se tomó en cuenta tipo de intervención, clasificación ASA o puntaje de riesgo anestésico y duración de la operación mayor al percentil 75.(1,5).

El seguimiento telefónico se hizo escogiendo al azar el $20 \%$ de los pacientes intervenidos y se llamaron a la casa entre el séptimo y doceavo día pos-evento. Durante los últimos seis meses de la muestra se llamaron al 100 $\%$, se les interrogó sobre signos de infección y quienes los presentaban o se tenía sospecha se citaron a consulta y valoración.

El procesamiento de los datos se hizo mediante el paquete estadístico EPIINFO 2002 y el análisis incluyó la estadística descriptiva, tomando porcentajes o promedios, media, desviación estándar, se construyeron tablas de frecuencia y gráficos, se compararon proporciones con la prueba chi cuadrado o estadístico $\mathrm{F}$ y las medias mediante el test de student, análisis de correlación y regresión múltiple, tomándose un nivel de confianza del 95\% con un valor $\mathrm{p}<0.05$.(8).

\section{RESULTADOS}

En total del período fueron 15625 pacientes con un promedio de edad de 48,7 años y D.E. +/-16,4 años, un rango de 3 a 95 años, moda de 42 y mediana de 49 años, hombres el 45,4 \% y mujeres 54,6 \%. El tiempo quirúrgico promedio fue de 61 minutos D.E. +/- 75, un rango entre 10 y 600 minutos y una mediana de 60 minutos. El número de cirugías que se hizo por especialidad fue: Cirugía general 5398 (35\%), oftalmología 4704 (30\%), cirugía plástica 1284 (8,2\%), Otorrino 1260 (8,1\%), Ortopedia 1204 (7,7\%), Urología 864 (5,5\%), Dermatología 143 (0,9\%) y otras 768 $(4,9 \%)$.

En la clasificación del tipo de herida se obtuvo: limpia 12128 (79 \%), limpia contaminada 3192 (21\%), contaminada $83(0,5 \%)$, y sucia $33(0,2$ $\%)$. Según el riesgo anestésico ASA, se clasificaron ASA cero 0,4 \%, ASA Uno $66 \%$, ASA dos $33 \%$ y ASA tres el $1 \%$, no hubo otros grupos, siendo de bajo riesgo nuestros pacientes.

Al aplicar el método de predicción de SENIC, la variable cirugía abdominal fue la más frecuente y de mayor sensibilidad. En el método NNISS, el mejor predictor podría ser cirugía mayor de dos horas, sin embargo sumados todos su sensibilidad es baja en nuestra población. La presencia de infección arrojó los siguientes riesgos relativos, intervalos de confianza y valor $\mathrm{p}$. Cirugía abdominal RR 1,14 (IC 1,04-1,26) y un valor p 0,0001; cirugía mayor 
de dos horas RR 1,27 (IC 1,06-1,52) valor p 0,0002; cirugía contaminada RR 1,81 (IC 1,03-3,2) valor p 0,0004; Asa 3,4,5 RR 1,36 (0,6-3,01) y valor $\mathrm{p}$ 0,26; no hubo pacientes con más de tres diagnósticos. El 15,8 \% presentaron tales factores.

Respecto al puntaje total obtenido en la escala SENIC, cero 13637 (88 $\%$ ) representaba pacientes sin ningún factor de riesgo, un factor 1693 (11 $\%)$, dos factores $150(0,96 \%)$, tres factores $9(0,06 \%)$ y el máximo sería cuatro, el cual lo presentó un solo paciente, esto ratifica que nuestra población de estudio es de bajo riesgo.

Durante el tiempo de seguimiento se detectaron 69 pacientes con infección de sitio quirúrgico, para una incidencia de 0,43 infectados por cada 100 pacientes intervenidos; es la segunda causa de infección nosocomial en nuestra institución con el 19,2\%. Según el tipo de herida: limpia $43(0,36$ $\%)$, limpia/contaminada 20 (0,63\%), contaminada 3 (3,6 \%) y sucia 3 (9 \%); no hubo diferencias entre grupos de edad teniendo un rango entre 17 y 86 años, en cambio por sexo, la infección estuvo presente en 21 (30,4 \%) hombres y en 48 (69,6 \%) mujeres, con un chi cuadrado de 6,27 y un valor $\mathrm{p}<$ 0.01 .

De acuerdo al tiempo quirúrgico, la cirugía con menor tiempo de intervención e infección fue de 20 minutos y la de mayor 360 minutos, entre treinta y noventa minutos se presentaron 38 casos, 21 de ellos entre los sesenta y noventa minutos. Cirugías con una duración mayor de dos horas e infección fueron 13, el análisis de regresión lineal muestra un estadístico $\mathrm{F}$ 17,61 y un valor $\mathrm{p}<0,000031$, siendo significativa la asociación tiempo e infección.

Según la clasificación SENIC ,entre los infectados hubo 22 pacientes a los cuales se les había practicado cirugía abdominal para una proporción de $1,46 \%$, cirugía mayor de dos horas 13 (3,09\%), contaminada 6 (5,5\%) y no hubo infección en personas con más de tres diagnósticos, el 39 \% tenían algún factor de riesgo. En cuanto NNISS, asa 3,4,5 un paciente tuvo infección (0,65\%), herida contaminada 6 (5,7 \%) y mayor de dos horas 13 (3,2 \%), el 23 \% tenían algún factor de riesgo.

La incidencia por cien egresos de infección por especialidad fue de 0,67 en Cirugía General, Oftalmología 0,27, Cirugía Plástica 0,31, Otorrino 0,08, Ortopedia 0,5 y Urología 0,58. Según el tipo de intervención e infección las más frecuentes fueron: extracción del cristalino y colocación de lente intraocular 8 (11,6 \%), Colecistectomía 6 (8,7\%), Varicosafenectomía 5 (7,2 \%), 
pterigio 3 (4,3\%), inyección vitreo 2 (2,9 \%), eventrorrafia 2, mamoplastia 2, lipoma 2 y mama supernumeraria 2 , entre otras.

El aislamiento de gérmenes se logró en 47 casos, siendo los más frecuentes en su orden: E. coli (14,9\%), Pseudomona aeruginosa (10,6 \%) y Staphilococcus epidermidis (10,6 \%), Staphilococcus aureus (8,5 \%), Serratia marcencens (8,5 \%), Morganella morgagni (8,5\%), Staphilococcus sciuri (6,4\%), Staphilococcus haemolyticus (4,26 \%), Klebsiella ornithiolytica (4,26 \%), Cedecea lapager (4,26 \%) y Proteus mirabilis (4,26\%).

\section{DISCUSIÓN}

En nuestro estudio encontramos una proporción de infección de sitio quirúrgico del 0,45 \% que es baja comparativamente, sin embargo nuestra institución es de mediana y baja complejidad, con una prevalencia calculada del 5 $\%$ en datos fragmentarios de la Secretaria de Salud de Bogotá, no existen otros puntos de referencia. Se han publicado infinidad de estudios en el mundo sobre infección de sitio quirúrgico, pero entre los datos más completos se encuentran los del NNISS, con un seguimiento de 1986 a 1996 a 593 344 procedimientos quirúrgicos, encontrándose 15523 con infección del sitio operatorio, que correspondía al 2,6\%. Entre los pacientes quirúrgicos, la literatura reporta que la infección del sitio operatorio fue la más frecuente de las infecciones nosocomiales (38 \%); de éstas, dos terceras partes se limitaban a la incisión, mientras un tercio involucraba órganos y espacios implicados en la cirugía. $(1,6,9)$.

Tabla 1. Proporción comparativa de infección de sitio quirúrgico,

\begin{tabular}{|c|c|c|c|c|}
\hline Clasificación & $\begin{array}{c}\text { Proporción } \\
\text { Mundial }\end{array}$ & $\begin{array}{c}\text { Fundación } \\
\text { Santa fe }\end{array}$ & $\begin{array}{c}\text { Clínica } \\
\text { Lleras-ISS }\end{array}$ & NNISS \\
\hline Jimnia & & & 0,3 & 2 \\
\hline 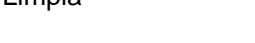 & 2,10 & 1,14 & 0,63 & 3,7 \\
\hline Limpia contaminada & 3,30 & 3,22 & & \\
\hline Contaminada & 6,40 & 10,9 & 3,60 & 9,2 \\
\hline Sucia & 7 & 23 & 9,00 & 23,1 \\
\hline
\end{tabular}

El estudio más cercano es de la Fundación Santafe, realizado a finales de 1999 en 2853 pacientes. La gran mayoría de las heridas quirúrgicas pertenecientes al programa corresponden a intervenciones clasificadas como limpias, 70,6 \%, seguidas de las heridas limpias-contaminadas, un $26,1 \%$ y las restantes pertenecen a procedimientos contaminados con un 3,3 \% (10). Nosotros tenemos una casuística en proporción similar, limpia 78,6 \%, limpia 
contaminada un 20,7 \% y contaminadas el 0,75 \% $(11,12)$. En la siguiente tabla se muestra la prevalencia según el tipo de herida comparativa.

En un estudio reciente Delgado-Rodríguez mostró que los criterios NNISS son más discriminantes que los SENIC; además que el NNISS es útil para predecir incluso la incidencia de neumonía postoperatoria (13). En nuestro estudio, la escala de mejor aplicación y sensibilidad es la SENIC debido a la presencia de la variable cirugía abdominal, frecuente evento quirúrgico y con un peso en la infección de sitio quirúrgico importante, encontrándose 22 casos; en las instituciones de mediana a baja complejidad el ASA o riesgo anestésico de los pacientes intervenidos es menor, componente de la escala NNISS. El porcentaje de personas detectadas con riesgos por la escala SENIC fue $39 \%$ mientras que en la escala NNISS fue de $23 \%$. En cuanto a la variable tiempo quirúrgico, para nosotros es más sensible entre 50 y 120 minutos, y no la aplicación del criterio mayor de dos horas, ya que son pocas las intervenciones que sobrepasan ese tiempo. El promedio total fue de 61 minutos, pero entre los infectados fue de 90 minutos, lo que arroja un estadístico $\mathrm{F}$ de 9,26 y un valor $\mathrm{p}<0.002$. Teniendo en cuenta el puntaje total en cada escala y los infectados, contamos con la siguiente proporción según el número de factores de riesgo (Tabla 2).

Tabla 2. Proporción de infección según puntaje total escalas SENIC, NNISS y comparación con CDC Atlanta

\begin{tabular}{|c|c|c|c|c|c|c|c|c|}
\hline & & & & & & & \\
\hline \multicolumn{4}{|c|}{ SENIC } & \multicolumn{4}{|c|}{ NNIS } & \multirow{2}{*}{$\begin{array}{c}\text { CDC } \\
\text { Atlanta } \\
\text { SENIC/ NNIS }\end{array}$} \\
\hline $\begin{array}{c}\text { Factores } \\
\text { riesgo }\end{array}$ & Pacientes & Infectados & $\%$ & $\begin{array}{c}\text { Factores } \\
\text { riesgo }\end{array}$ & Pacientes & Infectados & $\%$ & \\
\hline 0 & 13637 & 42 & 0,3 & 0 & 14822 & 53 & 0,36 & $1 / 1$ \\
\hline 1 & 1693 & 15 & 0,89 & 1 & 601 & 13 & 2,16 & $3,6 / 3$ \\
\hline 2 & 150 & 2 & 6,7 & 2 & 25 & 2 & 8 & 17 \\
\hline 3 & 9 & 3 & 22 & 3 & 5 & 1 & 20 & $17 / 15$ \\
\hline 4 & 1 & - & - & - & - & - & - & $27 \quad /$ \\
\hline
\end{tabular}

En cuanto a la microbiología de la infección de la herida quirúrgica los gérmenes más frecuentemente implicados en la infección del sitio operatorio, según los reportes de la literatura continúan siendo los mismos que en años anteriores; en primer lugar, la Escherichia Coli con 22,3 \% de incidencia; Staphylococcus aureus, 14,1 \%; Enterococcus faecalis con 10,5 $\%$, seguido de Klebsiella pneumoniae y Staphylococcus epidermidis con 8,2 $\%$, respectivamente. En el $43 \%$ de las heridas cultivadas se evidenciaron infecciones poli microbianas y en 2 heridas se aislaron gérmenes inusuales como la Cándida parapsilosis y el bacilo antracis $(6,14)$. En el estudio de la Fundación Santafe, los gérmenes más frecuentemente encontrados fueron: Staphylococcus coagulasa (-) y Escherichia coli con 29,2 \%, respectivamente, seguidos de Enterococcus faecalis con 17,6 \%; 
Staphylococcus aureus con 12,6 \%; Klebsiella pneumoniae con 4,8 \%; y Pseudomona aeruginosa con 4,6 \%.(10). Otras series muestran una prevalencia de 3,9 \% y los gérmenes asociados fueron: Staphylococcus coagulasa negativo, 28,4\%, enterococcus; $17,3 \%$, candida, 7,9 \% y Staphylococcus aureus, 8,7 \% (6). Otros han confirmado por lo menos el 65 $\%$ de la infección quirúrgica nosocomial, encontrando enterococcus $28 \%$, Staphylococcus aureus, $13 \%$, pseudomona, $11 \%$ y Cándida, $10 \%$, siendo superficiales el $24 \%$ y profundas el $4 \%$, polimicrobianas el $41 \%$, y $17 \%$ no fueron cultivadas. Correspondieron a cirugía general el $50 \%$, transplante $34 \%$ y cirugía cardiovascular el 15,8 \% (6,7). En nuestro estudio los gérmenes más frecuentemente aislados fueron E. coli, Pseudomona Aeruginosa y Staphylococcus de diferentes especies, concordante con los hallazgos descritos en la literatura. El aislamiento de gérmenes se hizo en su orden en los siguientes servicios: cirugía general $52 \%$, oftalmología 19,8 \%, Ortopedia 8,7 \%, Urología 7,2 \%, plástica 5,8 \% y otorrino 1,4 \%.

En países desarrollados el programa de vigilancia ha disminuido la utilización de profilaxis innecesaria, la estancia preoperatoria, la infección de 18,4 a 14 x 1000 días paciente y a una tasa global de 0,56; mientras que al parecer la infección de sitio quirúrgico, urinaria y pulmonar permanece sin cambios (13). En nuestra institución en 1998 se tenía una incidencia de 8,6 x 1000 días estancia y en el 2002, 13,3 x 1000 días estancia, variación probablemente debida a la mejoría del registro por la vigilancia intensificada, así como 2,9 x 100 egresos en el 2002; sin embargo, la incidencia general de infección nosocomial se ha reducido. La disminución de la infección posquirúrgica debido a todos estos componentes en nuestra institución se ha determinado en un $25 \%(14,15)$.

\section{CONCLUSIONES}

El desarrollo de infección es un proceso dinámico entre bacterias, huésped y el medio ambiente, interacción que se debe conocer y estudiar en todos los ámbitos hospitalarios. La infección de sitio quirúrgico ocupa los primeros lugares en frecuencia de presentación en las instituciones de salud generando un alto costo no solo económico sino en vidas. En nuestra institución, ocupa el segundo lugar, luego de la infección asociada a la atención del parto. Siendo este un evento prevenible, por ende susceptible de disminuir, se implementó un sistema de vigilancia intensificada que ha surtido benéficos efectos a la institución y sus usuarios identificando los factores de riesgo, generando medidas de prevención en pacientes que por su edad, enfermedades preexistentes y factores nutricionales, podrían tener mayor susceptibilidad a complicaciones; disminuyendo así los eventos mórbidos, lar- 
gas estancias hospitalarias, y alteración de la calidad de vida de los pacientes.

Consideramos que los hallazgos de nuestro estudio son relevantes y se pueden aplicar a todas las instituciones hospitalarias del país, aplicando las escalas de riesgo descritas SENIC-NNISS, siguiendo la misma metodología y en las instituciones de segundo nivel donde la complejidad de las intervenciones es menor y el tiempo quirúrgico más breve, recomendamos la aplicación de las escalas con una modificación en la variable tiempo, tomando el valor de sesenta minutos o como lo define el NNISS, percentil 75 del tiempo de cada institución. En cualquier circunstancia, la incidencia de infección es baja en forma significativa frente a lo reportado por la literatura. La nueva escala sugerida a implementar en nuestros pacientes en Colombia tendría las variables que se indican en la Tabla 3.

Tabla 3. Escala modificada SENIC en pacientes colombianos

\begin{tabular}{lccc}
\hline \multicolumn{1}{c}{ Variable } & SENIC & NNIS & $\begin{array}{c}\text { SENIC Modificada } \\
\text { Colombia Institución } \\
\text { segundo nivel }\end{array}$ \\
\hline Herida contaminada & $\mathrm{SI}$ & $\mathrm{SI}$ & $\mathrm{SI}$ \\
Cirugía abdominal & $\mathrm{SI}$ & $\mathrm{NO}$ & $\mathrm{SI}$ \\
Duración & 2 Horas & $>2$ Horas & $>$ de 60 minutos \\
Mas de tres Diagnósticos & $\mathrm{SI}$ & $\mathrm{NO}$ & $\mathrm{NO}$ \\
Asa 3,4,5 & $\mathrm{NO}$ & $\mathrm{SI}$ & $\mathrm{NO}$ \\
Sexo femenino & $\mathrm{NO}$ & $\mathrm{NO}$ & $\mathrm{SI}$ \\
\hline
\end{tabular}

El presente estudio tiene como limitaciones el hecho de seguir inicialmente al $20 \%$ de los pacientes intervenidos, aunque seleccionados al azar. Pudo dejar excluidos a pacientes infectados que hubieran consultado a otra institución. Similar efecto pudo tener la vinculación de pacientes en los primeros doce días pos evento, ya que un grupo pequeño de infección posquirúrgica se presenta después de dos semanas •

Agradecimientos. Al personal auxiliar de enfermería, sección de epidemiología. Clínica Carlos Lleras Restrepo: Norma Porras, Olga Velásquez y Roció Zabala.

\section{REFERENCIAS}

1. Malangoni M. Surgical site Infections: The cotting edge. Cuurrent opinion in infectious diseases. 1998; 11(4):465-469. 
2. Study efficacy of nosocomial infection control (SENIC)[Internet]: the nacional nosocomial infection surveillance Systems (NNISS) predictor of risk. Disponible en: http://www.cdc.org.gov.

3. Meakins J. Surgical infections diagnosis and treatment. Dsc. 1994: 127-137.

4. Perencevich E. Health and economic impact of surgical site infections diagnosed after hospital discharge. Emerging Infectious Diseases. 2003;9(2): 196-202.

5. Heinzelmam M, Melanie S, Lam T. Factors predisposing to bacterial invasion and infection. The americam journal of surgery. 2002;183(2):179-190.

6. Weis C. Six years of surgical wound infection surveillance at a tertiary care center: Review of the microbiologic and epidemiological aspects of 20.007 wounds. Archives of surgery. 1999;134(10):1041-1048.

7. Smith E, Emerson M. Geography is destiny: global nosocomial infection control. COID 2000;13(4):371-375.

8. Trapo R. Beth Dawson-Saunders. Bioestadística médica;1995.

9. Secretaria Distrital de Salud, Bogotá, D.C. Boletín Epidemiológico Distrital. Volumen 6 Nos 9,10 y 11; Agosto - noviembre 2001.

10. Domínguez AM [Internet]. Programa de Seguimiento de la herida quirúrgica y el sitio operatorio. Fundación Santafe de Bogotá 1989-1999.Disponible en: http:// www.fundacionsantafe.com. Consultado Marzo 2003.

11. Eslava J, Saavedra C, Cuervo S, Quemba J. Infeccion de sitio quirúrgico: factores relacionados en una institución de segundo nivel de atención. Infectio, 2002; 6 (2): 95.

12. Eslava J, Saavedra C, Cardona G, Quemba J. Vigilancia epidemiológica de infecciones intrahospitalarias en la Clínica Carlos Lleras Restrepo del seguro social. Boletín Epidemiológico Distrital. 2001;6 (7,8):7-11.

13. Delgado-Rodríguez. Efficacy of surveillance in nosocomial infection control in a surgical source. Americam Journal of Infection control. 2001;29(5):289294.

14. Mangram A. Guideline for prevention of surgical site infection. The hospital infection control practices advisory committee.1999; 265-266.

15. Vandenbroucke-Grauls C, Schultzs C. Surveillance in infection control: are we making progress? Curr. Opinion in Infect Dis.2002;15(4): 415-419. 